\title{
The Link between Aggrecan and Familial Osteochondritis Dissecans
}

\author{
Samantha Ozere $^{1}$, Sami Chergui ${ }^{2} \mathbb{D}$, Megan E. Cooke ${ }^{1} \mathbb{D}$, Thierry Pauyo ${ }^{2,3, *}$ and Derek H. Rosenzweig ${ }^{1,3, *(\mathbb{D})}$ \\ 1 Department of Surgery, Division of Orthopaedic Surgery, McGill University, Montreal, QC H3A 0G4, Canada; \\ samantha.ozere@mail.mcgill.ca (S.O.); megan.cooke.research@gmail.com (M.E.C.) \\ 2 Shriners Hospital for Children (Canada), Montréal, QC H4A 0A9, Canada; sami.chergui@mail.mcgill.ca \\ 3 Injury, Repair and Recovery Program, The Research Institute of McGill University Health Centre, \\ Montreal, QC H4A 3J1, Canada \\ * Correspondence: thierry.pauyo@mcgill.ca (T.P.); derek.rosenzweig@mcgill.ca (D.H.R.); \\ Tel.: +1-514-934-1934 (ext. 43238)
}

Citation: Ozere, S.; Chergui, S.; Cooke, M.E.; Pauyo, T.; Rosenzweig, D.H. The Link between Aggrecan and Familial Osteochondritis Dissecans. Surgeries 2021, 2, 128-138. https:// doi.org/10.3390/surgeries2020012

Academic Editor: Antonio Scarano

Received: 9 March 2021

Accepted: 21 March 2021

Published: 24 March 2021

Publisher's Note: MDPI stays neutral with regard to jurisdictional claims in published maps and institutional affiliations.

Copyright: (c) 2021 by the authors. Licensee MDPI, Basel, Switzerland. This article is an open access article distributed under the terms and conditions of the Creative Commons Attribution (CC BY) license (https:// creativecommons.org/licenses/by/ $4.0 /)$.

\begin{abstract}
Osteochondritis dissecans (OCD) is a chronic disease of the articular cartilage characterized by focal lesions of subchondral bone and overlaying cartilage. Through the growing number of reports describing the high prevalence of OCD in some families, the subcategory termed familial OCD (FOCD) was established. With the development of genetic approaches such as genome-wide association studies and sequencing, aggrecan (ACAN) has been identified as one of the genes of interest associated with FOCD. Aggrecan is a crucial protein for the preservation and function of cartilage. However, due to FOCD being characterized relatively recently, there is a paucity of literature on the subject. The purpose of this review is to explore the relationship between ACAN mutations and familial OCD as well as to explore current treatment options and avenues for future research. In vitro and animal studies have shown the importance of ACAN in the preservation of cartilage. However, the only human ACAN mutation related to OCD ever identified is a V2303M mutation in the G3 domain. Multiple treatments have been superficially explored, and some options such as growth hormone $(\mathrm{GH})$ and gonadotrophin-releasing hormone agonists (GnRHa) show potential. Thus, further research on FOCD in needed to identify other ACAN mutations and determine optimal treatment modalities for this patient population.
\end{abstract}

Keywords: cartilage repair; osteochondritis dissecans; aggrecan; surgery; treatments; orthopaedics; mutations; novel treatments; disease modelling

\section{Introduction}

Articular cartilage is an avascular connective tissue that covers bony surfaces in diarthrodial joints [1-4]. It has two main roles: to reduce friction between articular surfaces and to transfer loads to the underlying subchondral bone. These roles are facilitated by the extracellular matrix (ECM), which is composed of collagen type II and proteoglycans secreted by chondrocytes, the sole cell type in cartilage [3]. Osteochondritis dissecans (OCD) is a chronic disease of the articular cartilage characterized by focal, idiopathic lesions of subchondral bone and the overlaying cartilage $[5,6]$. These lesions cause destabilization and separation of the articular cartilage from the subchondral bone. OCD typically presents clinically as the joint catching, locking, or giving way, alongside pain [3,7]. OCD affects the growth plate or physes of bones, most commonly in the knee, ankle, and elbow. The incidence of OCD is around 15-29 per 100,000 people [8]. If the growth plate is open when the lesion begins it is classified as juvenile OCD, otherwise it is considered as the adult form [9]. Left untreated, OCD lesions can lead to early-onset osteoarthritis (OA) [10].

$\mathrm{OA}$ is a leading cause of disability in older adults, often characterized by degeneration of the articular cartilage and subchondral bone [6]. According to the Osteoarthritis Research Society International (OARSI), OA is a chronic disease and the most common 
form of arthritis. It can affect all joints in the body, including the cartilage, bone, synovium, ligament, and muscle tissues whereby tissue degeneration and inflammation in severe cases can drive pain and loss of mobility. OA has a large socioeconomic impact across the world; in the knee alone it affects $10 \%$ of men and $13 \%$ of women over the age of 60 [11,12]. Treatments for OA are limited and, with increasing joint degradation, range from physiotherapy to strengthen the joint, non-steroidal anti-inflammatory drugs (NSAIDS) for pain, intraarticular steroid injections, arthroscopic lavage, and debridement and microfracture [2]. These surgeries provide minimal relief for advanced OA and often result in deposition of fibrocartilage, which is mechanically inferior to native hyaline cartilage [2]. Total knee arthroplasty (TKA) can provide more relief and restore joint function. However, arthroplasty is an extremely invasive surgery and implants typically last for only 15-20 years depending on the properties of the replacement material [2,7]. Revision surgeries are challenging and limited, so joint replacement is not recommended for younger patients [13]. Briefly, OCD lesions in children and young adults must be treated quickly and effectively to prevent early-onset $\mathrm{OA}$.

Treatments for OCD depend on the severity of the lesion, fragment location, and status of the growth plate. If the lesion is stable and not likely to be displaced, which is common in early stages of the disease, non-operative interventions such as activity modification and reduced weight bearing can be applied [10,14]. Lesions in juvenile OCD patients have been shown to be much more likely to spontaneously heal compared to those with the adult form [15-18]. Similar to OA arthroscopic lavage, removal of loose bodies and debridement are among the less invasive treatment options that aim to stabilize lesions, preventing further deterioration of the cartilage surface [7]. Bone marrow stimulation is also a common technique that aims to recruit pluripotential stem cells from the bone marrow in order to help heal the cartilage lesion. Techniques, also used in OA, include microfracture, which aims to harness the capacity for cartilage to self-repair [2,3]. These techniques tend to regenerate fibrocartilage as well as assist in stabilization of the subchondral bone and the loose fragment $[18,19]$. Rigid fixation using rods, screws, wires, or bone pegs to fix the fragment in place are used to restore the subchondral plate and help heal the lesion [7]. More invasive surgical procedures include autologous chondrocyte implantation (ACI) and mosaicplasty, where cylindrical osteochondral grafts are transferred from minimally weight bearing regions of the joint to regenerate full thickness hyaline cartilage [2,7]. All techniques aim to restore joint function, reduce pain, and prevent further joint deterioration.

The exact etiology of the disease is currently unknown. Multiple theories have been proposed but none are able to explain the occurrence of OCD. Factors that have shown to play a role include repeated mechanical trauma, ischemia, abnormal ossification, ligament laxity, and genetic predisposition [20]. Findings show that $55-60 \%$ of OCD patients participate in regular athletic activities, which supports the theory that repetitive mechanical trauma leads to the progression of the disease [3]. However, it is thought that the cause of the disease may be multifactorial and a combination of factors is likely responsible. OCD sometimes presents in multiple members of the same family, which points to a genetic link in a variant of OCD [21]. This has led to the development of the subcategory familial OCD (FOCD). FOCD has been linked to short stature, advanced bone age, and early-onset osteoarthritis (OA) [21-23]. The genetic etiology of FOCD is yet to be fully understood, as is the role it plays in the larger picture of OCD, but recent studies have identified specific genes with causative mutations.

Various gene mutations have been identified as potential cascade triggers in animals who present with OCD similar to that of humans. Most of the genes identified are involved in matrix production, growth plate maturation, endochondral ossification, or cellular signaling $[24,25]$. Some examples of possible genetic causes in animals are parathyroid hormone 1 receptor (PTH1R), collagen type III alpha 1 chain (COL3A1), or UDP-glucose dehydrogenase (UGDH) [20]. While possible associations between certain genes and FOCD have been shown in animal models, there is still an important lack of literature relating to human patients [24]. The only gene to have been documented in human cases of FOCD is 
the aggrecan (ACAN) gene. However, despite being the gene of greatest interest in humans for OCD, there is a scarcity of literature on the phenotypical manifestations of ACAN mutations. Consequently, the pathway relating ACAN to FOCD is still poorly understood, and no specific treatment guidelines have been established. Currently, there is very little research on the treatment of patients with FOCD due to its rarity. Patients are generally treated in the same manner as any other OCD lesion without addressing the underlying cause of the genetic condition, leaving patients at risk of further lesions.

This review aims to explore the relationship between ACAN mutations and familial OCD. We first highlight the treatment modalities used in familial OCD and examine how they relate to the treatment of OCD. We then explore the potential for future research and how it may help to better understand other orthopedic conditions.

\section{Aggrecanopathies and OCD}

\subsection{Role of Aggrecan in Normal Physiology}

The cartilage extracellular matrix (ECM) produced by chondrocytes contains collagen type II, proteoglycans, hyaluronic acid (HA), glycoproteins, and other elastic fibers [26]. Proteoglycans are proteins that are covalently bound to anionic glycosaminoglycans (GAG) side chains [27]. About $10 \%$ of cartilage ECM is made of proteoglycans, and aggrecan is the proteoglycan in greatest abundance [26]. Aggrecan is composed of three globular domains named G1, G2, and G3 and binds with HA [4,26,28]. Aggrecan also contains three extended domains: the inter-globular domain (IGD), the keratan sulfate domain (KS), and the chondroitin sulfate domain (CS) (Figure 1) $[4,26,28]$.

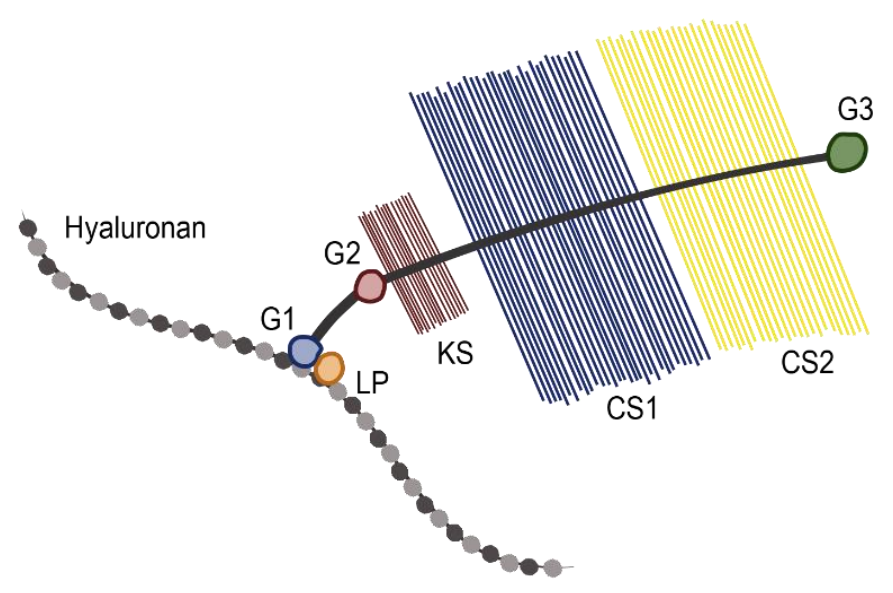

Figure 1. Structure of Aggrecan. The main protein binds to the hyaluronan backbone through G1 and link protein (LP) domains. Keratin sulfate (KS) and chondroitin sulfate (CS1 and CS2) sulfated glycosaminoglycans, which yield the net negative charge responsible for retaining water content in the tissue, are depicted.

Aggrecan contributes to the impact absorption of the ECM by influencing the fluid pressurization of cartilage [4]. In other words, when aggrecan is optimally expressed (along with its GAG chains), cartilage in the joint functions optimally with smooth gliding motion, high water content, and maximal resistance to compressive mechanical forces. When present in high concentrations, the negatively charged anionic groups on the GAG chains of aggrecan attract many mobile cations in the cartilage. About $90 \%$ of aggrecan is composed of GAG chains, which are predominantly made of chondroitin sulfate chains [26]. Thus, the presence of these cations generates a large osmotic gradient that pulls water into the cartilaginous tissue. This redistribution of water causes the ECM to swell. This swelling force is balanced by tensile resisting forces of the collagen matrix. This balance is crucial for the mechanical properties of cartilage such as impact absorption. When subjected to compressive forces, aggrecan does not diffuse freely within the tissue because it is fixed in multi-molecular aggrecan complexes. Instead, the aggrecan molecules at the site of 
compression are brought closer together, increasing their swelling potential. When the compressive force is removed, swelling reoccurs and the meshwork returns to its original form as the equilibrium is restored [4,26]. In OA, degradation of aggrecan has been shown to reduce the mechanical properties of the tissue, attributed to less water being bound to the sulphated GAGs [29]. Degradation of aggrecan molecules by A Disintegrin and Metalloproteinase with Thrombospondin Motifs (ADAMTS) -4 and -5 has been shown to be responsible for matrix degradation in human OA, leading to a reduced ability for the tissue to resist compressive forces [30,31]. Aggrecan has also been shown to play a role in intercellular interactions between chondrocytes and in interactions between chondrocytes and the matrix [32]. Higher aggrecan concentrations were shown to correlate with increased amounts of link protein on the chondrocyte's cellular surface, as well as improving their survival in suspended culture [33]. In order for cartilage to perform its mechanical and biological functions, it is commonly agreed that aggrecan must be present in large concentrations in order for it to be able to form aggregates and to be sufficiently sulfated [4,29]. It is also therefore expected that, should aggrecan reduce in quantity or not function normally (such as in OA or in certain cases of osteochondritis dissecans), joint degeneration will ensue.

The G1 domain constitutes the amino terminus of the protein and interacts with HA [34]. This 100 amino-acid long region is encoded by exons 3, 4, 5, and 6 of ACAN [26]. Conversely, G2 is unable to interact with HA, and its function is still poorly understood. Two B-like domains form G2, encoded by exons 8, 9, and 10 of ACAN [26]. The three extended domains, IGD, KS, and CS, are encoded by exons 7, 11, and 12, respectively [26]. These three domains seem important for physiological turnover, distribution within tissues, and interactions with water [26,32]. G3 is made up of a complement regulatory protein-like module, a C-type lectin-like module, and two epidermal growth factor-like modules [26,34]. G3, encoded by exons 13 to 19 of ACAN, seems to have a role in the transport of aggrecan within the chondrocyte and for its secretion into the extracellular matrix [26]. G3 seems to also be important for binding to various ligands and for creating the aggrecan meshwork. Indeed, in the cartilage matrix, aggrecan exists as large aggregates where multiple molecules are non-covalently bound to central HA molecules [26].

\subsection{Diseases Associated with ACAN Mutations}

Aggrecan has been shown to be important for the structure and development of cartilage from research involving naturally occurring aggrecan mutations in embryonically lethal chicken (nanomelia) and cartilage matrix deficient mice (cmd) [28]. Human diseases associated with aggrecan mutations are relatively rare, the first being identified in 2005 [22]. A variety of diseases have since been associated with aggrecan and present with a broad phenotypic spectrum due to an allelic series of ACAN mutations [28,35]. Homozygosity in the G3 domain (p.Asp2267Asn) results in spondyloepimetaphyseal dysplasia (SEMD), aggrecan type [28,35]. SEMD is a condition found in a single family characterized by severe skeletal dysplasia with extreme short stature and craniofacial abnormalities [28]. It has been suggested that heterogenous carriers may have a mild short stature phenotype [28]. Heterozygous mutations result in less severe phenotypes, as found in Spondyloepiphyseal dysplasia Kimberley type (SEDK), and FOCD [28,36,37].

SED Kimberly is caused by a frameshift mutation on the variable region of exon 12 (c.3986dupC), which encodes for the sGAG region of the aggrecan molecule [28]. These patients present with short stature, severe osteoarthritis, and delayed bone age [28]. Idiopathic short stature (ISS) has also been likened to heterozygous mutations in the ACAN gene [23,28,35-37]. Patients have shown symptoms including delayed or advanced bone age and OA. So far, 40 families worldwide have been identified with varying ACAN mutations causing ISS [35]. 


\subsection{Genetic Link to OCD}

At the time where genetic testing was still in its early stages, the notion of OCD being influenced by genetic factors was subject to debate. Indeed, a study by Petrie et al. in 1977 observed the incidence of OCD in the first-degree relatives of OCD patients [38]. This study concluded that there was no genetic etiology behind the development of OCD [38]. However, various reports describing OCD lesions in monozygotic twins and reports of multigenerational familial cases of OCD strengthen the argument in favor of a genetic cause of some cases of OCD [20,39-41]. Gornitzky et al. reported that $13.6 \%$ of their OCD patients had an immediate or extended family member with OCD [17]. The prevalence of OCD in the examined families was much higher than the prevalence in the general population and was consequently seen as compelling evidence of a potential genetic cause to OCD [17].

With the development of genetic sequencing, multiple mutations in the ACAN gene have been associated with the various abnormalities of aggrecanopathies. However, only one study identified a specific ACAN mutation associated with familial OCD. A genomewide study was performed by Stattin et al. on a five-generation family with hereditary OCD in 57 family members [42]. Of the 57, 19 family members were affected, and all shared a $10.5 \mathrm{Mb}$ haplotype encompassing around 100 genes, including ACAN. Due to ACAN's established role in cartilage physiology, this gene was sequenced in the affected patients. The team observed that the family carried a heterozygous missense mutation in exon 17 (c.6907G > A), causing a V2303M amino acid substitution in the C-type lectin (CLD) module of the G3 domain in aggrecan. This mutation was found in all 19 affected individuals and was absent in all remaining members of that family without OCD [42]. The mutation was also absent in 230 control chromosomes in healthy individuals unrelated to the family of interest. The group also showed that this ACAN mutation demonstrated an autosomal-dominant pattern of inheritance in the family [42].

The mutation mentioned in the previous paragraph is located at one of the known ligand-interaction sites of G3, and modeling has also suggested that a V2303M mutation would cause a conformational change of the C-type lectin module [42]. This affected amino acid is also conserved amongst many species. Consequently, this specific valine can be thought to be crucial for the previously mentioned roles of G3. Mutant and wild-type aggrecan proteins were produced to compare the CLD's binding affinity to proteins it is known to interact with: fibulin-1, fibulin-2, and tenascin-R. Affinity testing showed that the mutated G3 domain had decreased or completely terminated binding to these three proteins, demonstrating that the V2303M mutation has an important effect on the domain's function [42]. The mutant aggrecan's decline in affinity with various proteins would explain the altered ECM formation that would lead to a predisposition to OCD and adjacent symptoms such as short stature.

Mass spectrometry showed that patients with a heterozygous V2303M mutation had similar wild-type and mutated aggrecan levels in the joint cartilage [42]. Consequently, the fact that a phenotype was manifested in these heterozygous patients indicates that the mutated proteins play a dominant-negative role. This is consistent with the genetic lineage of the family of interest, which shows an autosomal-dominant pattern.

The characterization of the various genetic causes of OCD is still in its early stages. More studies are required on various different families in order to identify other mutations in the ACAN gene that would also manifest as familial OCD.

\section{Current Research on Aggrecan Mutations}

\subsection{Natural Aggrecan Mutations in Animals}

Animal models for aggrecan gene mutations are limited because of aggrecan's essential role in skeletal maturation [28]. Natural knockout of the aggrecan gene have been observed in cartilage matrix deficient ( $\mathrm{cmd}$ ) mice and the nanomelic $(\mathrm{nm})$ chicks [28].

Homozygous mice are born with dwarfism, short snouts, cleft palates, and tightly packed chondrocytes and minimal matrix formation lacking in aggrecan [43]. The mice do not live long and die from respiratory failure after birth. Heterozygous mice are born 
seemingly normal but present with slight dwarfism that worsens over time, with late onset spinal misalignment developing one year after birth. Mice with spinal misalignments develop a spastic gate and become unable to eat, leading to death. With one allele of the aggrecan gene, it is suggested that metabolism of aggrecan can result in varying phenotype. However, knee and other cartilaginous tissues appear normal [43]. The link protein binds to hyaluronan and the G1 domain of aggrecan [43]. Link protein knockout mice show similar but somewhat increased mild symptoms. Heterogeneous mutants show no phenotypic changes compared to homogeneous mutants, who die shortly after birth and show moderate dwarfism. Delayed endochondral ossification has also been observed in homogeneous mice [43].

Homozygous recessive nanomelic (nm) chicks present a mutation in the aggrecan protein and show reduced head and trunk size [44]. They also exhibit short and malformed limbs, with a growth plate lacking matrix and zonal demarcation. They do not, however, show a reduced number of skeletal elements. This model showed dysregulation of genes critical to the maturation process of hypertrophic chondrocytes. This leads to a small and disorganized hypertrophic zone that overlaps with the pre-hypertrophic zone in the growth plate [44].

Equine osteochondrosis is an orthopedic disease in horses similar to OCD in humans [24]. It can lead to thickened cartilage regions, necrotic areas, synovial effusions, and loose fragments. This is caused by altered endochondral ossification with abnormal chondrocyte differentiation and maturation. Candidate gene analysis in a pure-bred population of Spanish horses identified three single nucleotide polymorphisms associated with the lesions located in the FAF1, FCN3, and COL1A2 genes [24]. There has been vast research in the equine field due to the economic impact caused by the lameness induced by the disease. However, no gene link in equine osteochondrosis has yet been associated with aggrecan [24]. Intense breeding in miniature Shetland ponies, selecting for small size and other traits, has supported dwarfism with significant skeletal abnormalities [45]. A dwarf miniature Shetland pony presents with shortened limbs along with malformed neck, skull, and torso. Through genome wide sequencing, the ACAN:g.94370258G >C mutation in exon 7 has been demonstrated [45].

\subsection{In Vitro Studies}

A study by Xu et al. used bone marrow mesenchymal stem cells (BM-MSC) that were isolated from a patient who was part of the same genome wide study by Stattin et al. mentioned previously, of a northern Swedish family with FOCD, which identified a heterogeneous mutation in aggrecan exon 17 [21,46]. These FOCD BM-MSCs cultured in vitro were shown to be morphologically similar to healthy BM-MSCs. The FOCD cells were cultured in pellets and induced to differentiate into chondrocytes using chondrogenic media with transforming growth factor-B3 (TGF-B3). Results showed that the FOCD BM-MSCs pellets formed an irregular discoid morphology compared to controls. Immunohistochemical staining of the pellets showed minimal aggrecan in the ECM for the FOCD BM-MSCs, suggesting irregular processing and stabilizing of aggrecan ECM. Confocal microscopy confirmed that the patient derived cells had an ECM lacking aggrecan, and it was concentrated within the cells around the endoplasmic reticulum, indicating that the FOCD BM-MSCs failed to assemble and secrete aggrecan after differentiation. The ECM of the FOCD patient showed a 50\% reduction of aggrecan proteins and upregulation of proteins associated with cartilage degeneration, showing a composition of ECM proteins similar to advanced OA [46]. The deficiency in aggrecan secretion from the endoplasmic reticulum likely explains why FOCD patients exhibit early onset OA. The cmd mice and the nm chick animal models show the importance of aggrecan in the ECM, as they both exhibit significant growth abnormalities $[43,44]$. Due to this, the irregular morphology in the FOCD pellets is expected with a low aggrecan in the ECM, indicating that aggrecan plays a significant role in stabilizing the structure of the ECM. 


\subsection{Case Reports and Treatment}

Multiple case reports have described families with multiple members being affected by OCD lesions [9,31,40,41,47-49]. Pain, swelling, locking, and functional impairment were common symptoms. Short stature was common amongst the affected families. The available pedigrees also all showed autosomal dominant patterns of inheritance. However, only two studies tested their patients to confirm an ACAN mutation. Ven der Steen et al. tested 29 short children from different families and identified four children with heterozygous ACAN mutations [50]. They noticed that, within their sample, midface hypoplasia, joint problems, and broad great toes are associated with ACAN mutations. They found that having at least two of these features alongside short stature and advanced bone age indicate a high probability of having an ACAN mutation. However, the detected mutation was not described and might be different from the one Stattin et al. detected in their genome-wide association study [42]. Consequently, the features described by Ven der Steen et al. also might not be associated with the mutation described by Stattin et al. Our team (Dr. Pauyo) has recently performed genetic testing and identified a novel heterozygous variant; NM_013227.3:c.5658delG, p.Phe1887Leufs*15 in the proband in a family with three affected individuals (study currently submitted and in review). This variant results in a single nucleotide deletion at the 5658 position on the complementary DNA, resulting in a frame-shift and premature stop codon. At the level of mRNA, the premature stop codon produces non-functioning mRNA that gets degraded, a process known as nonsensemediated decay. No protein is translated from this allele, and the cell survives with half the amount of normal protein that is produced from the normal copy of the Aggrecan gene (wild type). The remaining protein is insufficient to produce a normal phenotype, resulting in haploinsufficiency (study under review).

\subsubsection{Pharmacological Treatments}

Familial OCD has also been observed to be associated with osteoarthritis, even in skeletally immature patients $[8,17,21,39,48,51]$. Thus, it is important to explore treatment options to prevent further joint degradation. However, treatment modalities for patients with familial OCD due to ACAN mutations have not yet been properly explored. Treatment for only one patient with OCD due to ACAN has been reported. Stattin et al. observed that, amongst the 14 individuals with OCD in the same family, one male that was previously treated with growth hormone $(\mathrm{GH})$ during skeletal immaturity had significant height improvements [21]. His final height was $168 \mathrm{~cm}$, which was $11.5 \mathrm{~cm}$ greater than the average height of affected males in his family. The group consequently hypothesized that GH could be beneficial in familial OCD cases relating to ACAN.

Ven der Steen et al. also reported the use of GH in four children with ACAN mutations from three different families [50]. These four patients were treated with GH due to their short stature, but none of them had an OCD lesion. They were also treated with gonadotrophin-releasing hormone agonists (GnRHa) to postpone puberty and epiphyseal closure. The female patient achieved a final height of $145.5 \mathrm{~cm}$, which was $5 \mathrm{~cm}$ taller than her mother but 3.9 standard deviations below the Dutch national average. One of the male patients reached an adult height of $165.3 \mathrm{~cm}, 8 \mathrm{~cm}$ greater than his father but 2.6 standard deviations below the Dutch national average. The other two male patients measured $164.7 \mathrm{~cm}$ and $161.3 \mathrm{~cm}$ at final assessment but had not yet reached their adult heights. No adverse effects or decreased bone density were observed in the children. However, GnRHa had to be discontinued in the female patient due to a slowed growth rate. Thus, a combination of GH and GnRHa might be a beneficial treatment for aggrecanopathies resulting from different ACAN mutations.

\subsubsection{Conservative Treatments}

Conservative treatment has been reported in a few case reports, with variable results. Fonseca et al. treated two cousins suffering from familial OCD affecting the knee [5]. The first patient underwent casting and restriction of weightbearing for two months. A few 
months later, the lesion worsened radiographically, yet symptoms completely resolved with no limitations during physical activities. Twenty-one years later, the patient only experienced knee pain during intense physical activity. The other patient underwent the same treatment with an extra two months of weightbearing restriction and physical therapy. Seven years later, radiographs showed complete radiographic resolution of the lesion. The patient was also asymptomatic upon physical examination after 20 years. Others have reported that physical therapy, bracing, NSAID use, or activity restriction were not effective in their patients with familial OCD. Surgical intervention was necessary for those patients $[41,52]$.

\subsubsection{Surgical Treatments}

The use of surgical treatment has also been reported in cases of FOCD, but not in cases with a confirmed ACAN mutation. A few case reports have noted that fragment excision, drilling, and internal fixation can lead to pain relief and functional improvements [9,39,41,52,53]. Mackie et al. observed a pair of monozygotic twins for whom surgical debridement and loose body removal provided minimal pain relief [54]. They had both developed an osteochondral lesion in the medial femoral condyle of the left knee that required osteochondral allografting. Following surgery, both patients experienced complete pain relief and functional improvements, with Musculoskeletal Tumor Society (MSTS) scores of $100 \%$. Radiographic healing and graft incorporation were also reported post-operatively after 7 years for one twin and 5 years for the other.

The evidence supporting one particular treatment strategy for OCD related to ACAN mutations is still very weak. The literature has documented the treatment of only one patient with OCD and a confirmed ACAN mutation. All other case reports presented here regarding conservative or surgical treatments were centered on patients with familial OCD but no confirmed ACAN mutation. However, as the clinical presentation of the joint is similar, these treatments are likely to also be effective in patients with ACAN mutations.

\section{Future Perspectives}

It is possible to notice an important paucity in the literature relating to the involvement of ACAN in knee FOCD. Current research tends to focus on aggrecan relating to osteoarthritis or as an outcome measure for studies examining the efficacy of biologically active scaffolds $[52,53]$. As a result, there is a lack of knowledge regarding epidemiological data, identification of particular mutations in ACAN causing FOCD, prognostic factors, and preferred methods of treatment. More extensive exploration is required to propose confident recommendations for the management of these patients. The study of ACAN and its effects on the musculoskeletal system is a promising domain to research. Better comprehension of this crucial gene will yield a better understanding of FOCD as well as other debilitating conditions such as SEMD, SEDK, and related osteoarthritis.

Although there is limited literature on the relation of ACAN mutations in FOCD patients, there is a clear link between mutated ACAN and short stature, early onset OA, and skeletal abnormalities. Moreover, one genome-wide study has associated an ACAN mutation in 19 members of a five-generation family with FOCD, causing a substitution in the C-type lectin (CLD) module of the G3 domain of aggrecan [42]. An in vitro study using BM-MSCs from this same five generation family with FOCD showed a failure to assemble and secrete aggrecan from the ER, leading to a change in cartilage ECM proteins similar to that of advanced OA [46]. Only a handful of patients with ACAN mutations have been reported to have undergone treatment for their aggrecanopathies. GH and GnRHa have shown some promise as they have yielded improvements in terms of patient height. However, high quality studies are needed to assess their efficacy. Interestingly, visco-supplemetation has been shown to have some efficacy in treating adult OCD of the ankle joint [54], lowering pain. Alternately, cell-based therapy such as autologous chondrocyte implantation also showed clinical promise to repair lesions in juvenile OCD 
patients [55]. A major limitation for many of these trials is the lack of an appropriate in vitro or in vivo model system from which to study new OCD treatments.

A search through multiple international clinical trial databases has yielded only one experiment underway in the United States in which 30 pre-pubescent children with aggrecan deficiencies are administered somatropin (a form of growth hormone) [56]. Significant results from this trial would have an important clinical impact because growth hormone could be the option of choice for an early non-invasive intervention in pediatric patients suffering from aggrecanopathies such as FOCD. In summary, FOCD provides insight into the importance of aggrecan for the long-term functioning of articular cartilage and its role in OA. Understanding the genetic nature of FOCD can aid in developing therapeutic treatments that target aggrecan for OCD and osteoarthritis. Novel treatments that aim to restore and maintain the expression of aggrecan in the ECM could potentially reduce cartilage deterioration and in turn reduce the need for invasive surgeries. Further research on FOCD is required to determine the best possible treatment methods and preventative treatment for those with the aggrecan mutations. A more detailed genome wide study of patients with FOCD, juvenile OCD, and OCD is required to further understand the etiology.

While there have been several clinical studies and case reports over the years for various manifestations of OCD, there is still a large knowledge gap in understanding overall etiology and how best to improve treatments. This may be due to a lack of sophisticated in vitro models and a true animal model of OCD. The aforementioned osteochondrosis identified in several types of horses may be a candidate, but veterinary scientists may be more focused on ways to treat the animals for pain and improve mobility. There is little control, a high cost, and potential ethical issues associated with experimentation on such animals. Therefore, there is a current need for generating models of OCD. We propose that future studies should take advantage of induced pluripotent stem cell technology by converting OCD patient peripheral blood samples into stem cell lines. These in turn can be differentiated into osteoblastic and chondrogenic cells from which to model osteochondral interfaces. Biofabrication technology such as three-dimensional (3D) bioprinting can facilitate highly organized structures with a specific spatial cell distribution to mimic articular cartilage. These human "tissue-on-a-chip" can serve as highly useful screening tools to study matrix deposition and mechanics, and also screen novel therapeutics. These tools will also be highly useful for generating personalized medicine for those patients with familial OCD, where known genetic abnormalities can be targeted. With these improving technologies, the future may be much brighter for enhanced treatment of OCD.

Author Contributions: Conceptualization, D.H.R. and T.P.; writing—original draft preparation, S.O., S.C. and M.E.C.; writing-review and editing, S.O., S.C., M.E.C., T.P. and D.H.R.; supervision, T.P. and D.H.R. All authors have read and agreed to the published version of the manuscript.

Funding: Derek H. Rosenzweig acknowledges the Reseau De Recherche en Sante Buccodentaire et Osseuse (RSBO) for research support. Megan E. Cooke acknowledges funding from the Canadian Institutes of Health Research (Funding Ref. 171258) and the Research Institute of the McGill University Health Centre.

Institutional Review Board Statement: Not applicable.

Informed Consent Statement: Not applicable.

Data Availability Statement: Not applicable.

Conflicts of Interest: The authors declare no conflict of interest.

\section{References}

1. Handbook of Histology Methods for Bone and Cartilage; Humana Press: Totowa, NJ, USA, 2003.

2. Dzaja, I.; Khalid, S. Hip and knee osteoarthritis. In Osteoarthritis: Pathogenesis, Diagnosis, Available Treatments, Drug Safety, Regenerative and Precision Medicine; Springer: Cham, Switzerland, 2015; pp. 29-42. ISBN 9783319195605.

3. Krishnan, Y.; Grodzinsky, A.J. Cartilage diseases. Matrix Biol. 2018, 71-72, 51-69. [CrossRef] 
4. Roughley, P.J.; Mort, J.S. The role of aggrecan in normal and osteoarthritic cartilage. J. Exp. Orthop. 2014, 1, 1-11. [CrossRef] [PubMed]

5. Fonseca, A.; Keret, D.; Macewen, G. Familial Osteochondritis Dissecans. Orthopedics 1990, 13, 1259-1262.

6. Grässel, S.; Aszódi, A. Cartilage: Pathophysiology; Springer International Publishing: Berlin/Heidelberg, Germany, 2017; Volume 2, ISBN 9783319458038.

7. Cole, B.J.; Malek, M.M. Articular Cartilage Lesions; Springer: New York, NY, USA, 2004.

8. Andriolo, L.; Crawford, D.C.; Reale, D.; Zaffagnini, S.; Candrian, C.; Cavicchioli, A.; Filardo, G. Osteochondritis Dissecans of the Knee: Etiology and Pathogenetic Mechanisms. A Systematic Review. Cartilage 2020, 11, 273-290. [CrossRef] [PubMed]

9. Kozlowski, K.; Middleton, R. Familial osteochondritis dissecans: A dysplasia of articular cartilage? Skelet. Radiol. 1985, 13, 207-210. [CrossRef] [PubMed]

10. Bruns, J.; Werner, M.; Habermann, C. Osteochondritis Dissecans: Etiology, Pathology, and Imaging with a Special Focus on the Knee Joint. Cartilage 2018, 9, 346-362. [CrossRef] [PubMed]

11. Zhang, Y.; Jordan, J.M. Epidemiology of osteoarthritis. Clin. Geriatr. Med. 2010, 26, 355-369. [CrossRef] [PubMed]

12. Goldring, M.B.; Culley, K.L.; Otero, M. Pathogenesis of osteoarthritis in general. In Cartilage: Pathophysiology; Springer International Publishing: Cham, Switzerland, 2017; Volume 2, pp. 1-25. ISBN 9783319458038.

13. Grässel, S.; Aszódi, A. Cartilage: Physiology and Development; Springer International Publishing: Berlin/Heidelberg, Germany, 2016; Volume 1, ISBN 9783319295688.

14. Krakow, D. Heritable Diseases of Connective Tissue. In Kelley and Firestein's Textbook of Rheumatology; Elsevier: Amsterdam, The Netherlands, 2017; pp. 1797-1815.

15. Laor, T.; Zbojniewicz, A.M.; Eismann, E.A.; Wall, E.J. Juvenile osteochondritis dissecans: Is it a growth disturbance of the secondary physis of the epiphysis? Am. J. Roentgenol. 2012, 199, 1121-1128. [CrossRef] [PubMed]

16. Olstad, K.; Shea, K.G.; Cannamela, P.C.; Polousky, J.D.; Ekman, S.; Ytrehus, B.; Carlson, C.S. Juvenile osteochondritis dissecans of the knee is a result of failure of the blood supply to growth cartilage and osteochondrosis. Osteoarthr. Cartil. 2018, 26, 1691-1698. [CrossRef]

17. Gornitzky, A.L.; Mistovich, R.J.; Atuahuene, B.; Storey, E.P.; Ganley, T.J. Osteochondritis Dissecans Lesions in Family Members: Does a Positive Family History Impact Phenotypic Potency? Clin. Orthop. Relat. Res. 2017, 475, 1573-1580. [CrossRef]

18. Polousky, J.D. Juvenile osteochondritis dissecans. Sports Med. Arthrosc. 2011, 19, 56-63. [CrossRef] [PubMed]

19. van Weeren, P.R.; Olstad, K. Pathogenesis of osteochondrosis dissecans: How does this translate to management of the clinical case? Equine Vet. Educ. 2016, 28, 155-166. [CrossRef]

20. Gans, I.; Grant, S.F.A.; Ganley, T.J. The Genetic Nature of Osteochondritis Dissecans: A Systematic Review and Call for Improved Studies. Univ. Pa. Orthop. J. 2013, 23, 14-16.

21. Stattin, E.L.; Tegner, Y.; Domellöf, M.; Dahl, N. Familial osteochondritis dissecans associated with early osteoarthritis and disproportionate short stature. Osteoarthr. Cartil. 2008, 16, 890-896. [CrossRef] [PubMed]

22. Sentchordi-Montane, L.; Aza-Carmona, M.; Benito-Sanz, S.; Barreda-Bonis, A.C.; Sanchez-Garre, C.; Prieto-Matos, P.; Ruiz-Ocana, P.; Lechuga-Sancho, A.; Carcavilla-Urqui, A.; Mulero-Collantes, I.; et al. Heterozygous aggrecan variants are associated with short stature and brachydactyly: Description of 16 probands and a review of the literature. Clin. Endocrinol. (Oxf.) 2018, 88, 820-829. [CrossRef]

23. Nilsson, O.; Guo, M.H.; Dunbar, N.; Popovic, J.; Flynn, D.; Jacobsen, C.; Lui, J.C.; Hirschhorn, J.N.; Baron, J.; Dauber, A. Short stature, accelerated bone maturation, and early growth cessation due to heterozygous aggrecan mutations. J. Clin. Endocrinol. Metab. 2014, 99, E1510-E1518. [CrossRef]

24. Sevane, N.; Dunner, S.; Boado, A.; Cañon, J. Candidate gene analysis of osteochondrosis in Spanish Purebred horses. Anim. Genet. 2016, 47, 570-578. [CrossRef] [PubMed]

25. Laverty, S.; Ionescu, M.; Marcoux, M.; Boure, L.; Doize, B.; Poole, A.R. Alterations in cartilage type-II procollagen and aggrecan contents in synovial fluid in equine osteochondrosis. J. Orthop. Res. 2000, 18, 399-405. [CrossRef] [PubMed]

26. Kiani, C.; Chen, L.; Wu, Y.J.; Yee, A.J.; Yang, B.B. Structure and function of aggrecan. Cell Res. 2002, 12, 19-32. [CrossRef]

27. Yanagishita, M. Function of proteoglycans in the extracellular matrix. Pathol. Int. 1993, 43, 283-293. [CrossRef]

28. Gibson, B.G.; Briggs, M.D. The aggrecanopathies; an evolving phenotypic spectrum of human genetic skeletal diseases. Orphanet J. Rare Dis. 2016, 11, 1-8. [CrossRef]

29. Cooke, M.E.; Lawless, B.M.; Jones, S.W.; Grover, L.M. Matrix degradation in osteoarthritis primes the superficial region of cartilage for mechanical damage. Acta Biomater. 2018, 78, 320-328. [CrossRef]

30. Baker, N.; Sharpe, P.; Culley, K.; Otero, M.; Bevan, D.; Newham, P.; Barker, W.; Clements, K.M.; Langham, C.J.; Goldring, M.B.; et al. Dual regulation of metalloproteinase expression in chondrocytes by Wnt-1-inducible signaling pathway protein 3/CCN6. Arthritis Rheum 2012, 64, 2289-2299. [CrossRef] [PubMed]

31. Caterson, B.; Flannery, C.R.; Hughes, C.E.; Little, C.B. Mechanisms involved in cartilage proteoglycan catabolism. Matrix Biol. 2000, 19, 333-344. [CrossRef]

32. Watanabe, H.; Yamada, Y.; Kimata, K. Roles of aggrecan, a large chondroitin sulfate proteoglycan, in cartilage structure and function. J. Biochem. 1998, 124, 687-693. [CrossRef]

33. Yang, B.B.; Zhang, Y.; Cao, L.; Yang, B.L. Aggrecan and link protein affect cell adhesion to culture plates and to type II collagen. Matrix Biol. 1998, 16, 541-561. [CrossRef] 
34. Cao, L.; Zhang, Y.; Yang, B.B. Expression of the G1 domain of aggrecan interferes with chondrocyte attachment and adhesion. Matrix Biol. 1998, 17, 379-392. [CrossRef]

35. Stavber, L.; Hovnik, T.; Kotnik, P.; Lovrečić, L.; Kovač, J.; Tesovnik, T.; Bertok, S.; Dovč, K.; Debeljak, M.; Battelino, T.; et al. High frequency of pathogenic ACAN variants including an intragenic deletion in selected individuals with short stature. Eur. J. Endocrinol. 2020, 182, 243-253. [CrossRef]

36. Tatsi, C.; Gkourogianni, A.; Mohnike, K.; DeArment, D.; Witchel, S.; Andrade, A.C.; Markello, T.C.; Baron, J.; Nilsson, O.; Jee, Y.H. Aggrecan mutations in nonfamilial short stature and short stature without accelerated skeletal maturation. J. Endocr. Soc. 2017, 1, 1006-1011. [CrossRef] [PubMed]

37. Gkourogianni, A.; Andrew, M.; Tyzinski, L.; Crocker, M.; Douglas, J.; Dunbar, N.; Fairchild, J.; Funari, M.F.A.A.; Heath, K.E.; Jorge, A.A.L.L.; et al. Clinical Characterization of Patients With Autosomal Dominant Short Stature due to Aggrecan Mutations. J. Clin. Endocrinol. Metab. 2017, 102, 460-469. [CrossRef]

38. Petrie, P. Aetiology of Osteochondritis Dissecans. Failure to Establish a Familial Background. J Bone Jt. Surg Br. 1977, 59, $366-367$. [CrossRef]

39. Mubarak, S.J.; Carroll, N.C. Familial Osteochondritis Dissecans of the Knee. Clin. Orthop. Relat. Res. 1979, 140, 131-136. [CrossRef]

40. Mei-Dan, O.; Mann, G.; Steinbacher, G.; Cugat, R.B.; Alvarez, P.D. Bilateral Osteochondritis Dissecans of the Knees in Monozygotic Twins: The Genetic Factor and Review of the Etiology. Am. J. Orthop. 2009, 38, E152-E155. [PubMed]

41. Richie, L.B.; Sytsma, M.J. Matching osteochondritis dissecans lesions in identical twin brothers. Orthopedics 2013, 36. [CrossRef]

42. Stattin, E.L.; Wiklund, F.; Lindblom, K.; Önnerfjord, P.; Jonsson, B.A.; Tegner, Y.; Sasaki, T.; Struglics, A.; Lohmander, S.; Dahl, N.; et al. A Missense Mutation in the Aggrecan C-type Lectin Domain Disrupts Extracellular Matrix Interactions and Causes Dominant Familial Osteochondritis Dissecans. Am. J. Hum. Genet. 2010, 86, 126-137. [CrossRef]

43. Watanabe, H.; Yamada, Y. Chondrodysplasia of gene knockout mice for aggrecan and link protein. Glycoconj. J. 2002, 19, 269-273. [CrossRef] [PubMed]

44. Domowicz, M.S.; Cortes, M.; Henry, J.G.; Schwartz, N.B. Aggrecan modulation of growth plate morphogenesis. Dev. Biol. 2009, 329, 242-257. [CrossRef]

45. Metzger, J.; Gast, A.C.; Schrimpf, R.; Rau, J.; Eikelberg, D.; Beineke, A.; Hellige, M.; Distl, O. Whole-genome sequencing reveals a potential causal mutation for dwarfism in the Miniature Shetland pony. Mamm. Genome 2017, 28, 143-151. [CrossRef]

46. Xu, M.; Stattin, E.-L.; Shaw, G.; Heinegard, D.; Sullivan, G.; Wilmut, I.; Colman, A.; Onnerfjord, P.; Khabut, A.; Aspberg, A.; et al. Chondrocytes Derived From Mesenchymal Stromal Cells and Induced Pluripotent Cells of Patients with Familial Osteochondritis Dissecans Exhibit an Endoplasmic Reticulum Stress Response and Defective Matrix Assembly. Stem. Cells Transl. Med. 2016, 5, 1171-1181. [CrossRef]

47. Crippa, M.; Giangiobbe, S.; Villa, R.; Bestetti, I.; De Filippis, T.; Fatti, L.; Taurino, J.; Larizza, L.; Persani, L.; Bellini, F.; et al. A balanced reciprocal translocation $\mathrm{t}(10 ; 15)(\mathrm{q} 22.3 ; \mathrm{q} 26.1)$ interrupting ACAN gene in a family with proportionate short stature. J. Endocrinol. Invest. 2018, 41, 929-936. [CrossRef]

48. Andrew, T.A.; Spivey, J.; Lindebaum, R.H. Familial osteochondritis dissecans and dwarfism. Acta Orthop. 1981, 52, 519-523. [CrossRef]

49. Sato, K.; Moy, O.J.; Peimer, C.A.; Nakamura, T.; Howard, C.; Ko, S.H.; Lee, T.C.; Nishiwaki, Y. An experimental study on costal osteochondral graft. Osteoarthr. Cartil. 2012, 20, 172-183. [CrossRef] [PubMed]

50. van der Steen, M.; Pfundt, R.; Maas, S.J.W.H.; Bakker-van Waarde, W.M.; Odink, R.J.; Hokken-Koelega, A.C.S. ACAN gene mutations in short children born SGA and response to growth hormone treatment. J. Clin. Endocrinol. Metab. 2017, 102, 1458-1467. [CrossRef] [PubMed]

51. Muller, W.E.; Neufurth, M.; Wang, S.; Tolba, E.; Schroder, H.C.; Wang, X. Morphogenetically active scaffold for osteochondral repair (polyphosphate/alginate/N,O-carboxymethyl chitosan). Eur. Cell. Mater. 2016, 31, 174-190. [CrossRef] [PubMed]

52. Yellin, J.L.; Trocle, A.; Grant, S.F.; Hakonarson, H.; Shea, K.G.; Ganley, T.J. Candidate Loci are Revealed by an Initial Genome-wide Association Study of Juvenile Osteochondritis Dissecans. J. Pediatr. Orthop. 2017, 37, e32-e36. [CrossRef] [PubMed]

53. Reyes, R.; Delgado, A.; Sanchez, E.; Fernandez, A.; Hernandez, A.; Evora, C. Repair of an osteochondral defect by sustained delivery of BMP-2 or TGFbeta1 from a bilayered alginate-PLGA scaffold. J. Tissue Eng. Regen. Med. 2014, 8, 521-533. [CrossRef]

54. Mei-Dan, O.; Maoz, G.; Swartzon, M.; Onel, E.; Kish, B.; Nyska, M.; Mann, G. Treatment of Osteochondritis Dissecans of the Ankle with Hyaluronic Acid Injections: A Prospective Study. Foot Ankle Int. 2008, 29, 1171-1178. [CrossRef]

55. Beck, J.J.; Sugimoto, D.; Micheli, L. Sustained Results in Long-Term Follow-Up of Autologous Chondrocyte Implantation (ACI) for Distal Femur Juvenile Osteochondritis Dissecans (JOCD). Adv. Orthop. 2018, 2018. [CrossRef]

56. Growth Hormone Treatment in Patients With Aggrecan (ACAN) Deficiency-Full Text View—ClinicalTrials.gov. Available online: https: / / clinicaltrials.gov/ct2/show / NCT03288103?term=acan\%2C+aggrecan\&draw=2\&rank=1 (accessed on 9 March 2021). 